\title{
Möglichkeiten und Grenzen einer Zusammenarbeit mit Heilpraktikern aus ärztlicher Sicht
}

\author{
Mirjam Thanner ${ }^{\mathrm{a}} \quad$ Eckhard Nagel $^{\mathrm{a}} \quad$ Julika Loss $^{\mathrm{b}}$ \\ a Institut für Medizinmanagement und Gesundheitswissenschaften, Universität Bayreuth, \\ ${ }^{\mathrm{b}}$ Medizinische Soziologie, Institut für Epidemiologie und Präventivmedizin, Universität Regensburg, Deutschland
}

\section{Schlüsselwörter}

Heilpraktiker - Kooperation .

Komplementär- und Alternativmedizin

\section{Zusammenfassung}

Hintergrund: Deutschland weist gegenüber zahlreichen anderen Staaten die Besonderheit auf, dass neben Ärzten auch Heilpraktiker eine staatliche Zulassung zur Ausübung der Heilkunde erlangen können. Das Verhältnis der beiden Berufsgruppen zueinander gilt als schwierig - unabhängig von berufsrechtlichen Hindernissen. Ziel der Untersuchung war es zu klären, inwieweit bei niedergelassenen Ärzten eine Bereitschaft zur Zusammenarbeit mit Heilpraktikern besteht. Gleichzeitig sollten Bedingungen, Chancen sowie Hindernisse einer Kooperation aufgezeigt werden. Methoden: Um die ärztliche Bereitschaft zur Zusammenarbeit mit Heilpraktikern zu evaluieren, wurden mit 15 niedergelassenen Ärzten und Zahnärzten semi-strukturierte Interviews geführt. Die Fragen thematisierten Bedingungen, Chancen sowie Barrieren einer Kooperation. Im Anschluss wurden die Interviews wörtlich transkribiert und mithilfe der thematischen Inhaltsanalyse ausgewertet. Ergebnisse: Einer Zusammenarbeit gegenüber aufgeschlossene Ärzte sehen Heilpraktiker als ergänzende Therapeuten bei leichteren oder psychisch beeinflussten Erkrankungen. Dafür bestehen allerdings klare Bedingungen: Heilpraktiker müssten die Grenzen ihrer Behandlungsfähigkeit erkennen. Voraussetzungen seien auch transparente Regelungen der Kostenerstattung durch Krankenkassen, insbesondere dürften ärztliche Leistungen im Bereich der Komplementär- und Alternativmedizin nicht diskriminiert werden. Neben dem mangelnden Vertrauen der befragten Ärzte in die Heilpraktikerausbildung erweist es sich als hinderlich, wenn Heilpraktiker störend auf die bestehende Arzt-Patienten-Beziehung einwirken bzw. den Abbruch einer schulmedizinischen Therapie veranlassen. Gleichzeitig wird die bestehende Patientenversorgung bereits als zu komplex für eine Kooperation eingeschätzt. Schlussfolgerungen: Um zu reproduzierbaren Ergebnissen zu gelangen, sind Untersuchungen mit quantitativem Forschungsdesign nötig. Auch sollte geklärt werden, inwieweit aus Sicht der Heilpraktiker eine Zusammenarbeit mit Ärzten wünschenswert ist. Der Abbau von Kommunikationsbarrieren ist nötig, damit Patienten offen mit ihrem Arzt über Heilpraktikerbesuche sprechen können. Das Wissen über Unterschiede in Ausbildung und Zulassung zum Arzt oder Heilpraktiker ist Grundvoraussetzung für eine informierte Entscheidung der Patienten.

\section{KARGER \\ Fax +497614520714 \\ Information@Karger.com}

www.karger.com (c) 2013 S. Karger GmbH, Freiburg

$1661-4119 / 13 / 0201-0023 \$ 38.00 / 0$

Accessible online at:

www.karger.com/fok

\section{Keywords}

Non-medically trained practitioners - Cooperation .

Complementary and alternative medicine

\section{Summary}

Cooperation between Physicians and Non-Medically

Trained Practitioners: Advantages and Obstacles

Background: In Germany, not only physicians are allowed to practice medicine but also non-medically trained practitioners, so-called Heilpraktiker, can obtain a licensure to treat patients. In contrast to cooperation between doctors, a division of tasks and responsibilities between non-medically trained practitioners and physicians seems to be difficult. The aim of the study was to clarify to what extent doctors in private practice are willing to cooperate with non-medically trained practitioners. At the same time, conditions, opportunities, and obstacles of cooperation are described. Methods: In order to evaluate the willingness to collaborate with non-medically trained practitioners, semi-structured interviews were conducted with 15 physicians and 2 dentists in an outpatient setting. The interviews further focused on conditions, advantages, and obstacles to cooperation. The interviews were transcribed literally and analyzed using qualitative content analysis. Results: The interviews showed that physicians partly are willing to cooperate with non-medically trained practitioners, but there were strict conditions and also some serious obstacles to cooperation. Doctors who are open-minded about collaboration consider non-medically trained practitioners as additional therapists for minor ailments. Non-medically trained practitioners would have to respect their limitations, and health insurances should launch transparent contracts for remuneration and reimbursement. Physicians showed low confidence in training programs for Heilpraktiker. Additionally, it was thought to be harmful when non-medically trained practitioners interfere with the physician-patient relationship and try to interrupt conventional treatment or medication. Conclusions: Quantitative research is necessary to quantify data and generalize results from the sample to the population of physicians. Further, the willingness of non-medically trained practitioners to collaborate with physicians should be evaluated. Physicians should try to reduce communication obstacles so that patients can speak frankly about consultations of non-medically trained practitioners. 


\section{Einleitung}

Deutschland weist gegenüber zahlreichen anderen Staaten die Besonderheit auf, dass neben Ärzten auch Heilpraktiker eine staatliche Zulassung zur berufsmäßigen Ausübung der Heilkunde erlangen können [1]. Rechtsgrundlage dafür ist das «Gesetz über die berufsmäßige Ausübung der Heilkunde ohne Bestallung» (Heilpraktikergesetz, HeilprG) aus dem Jahre 1939 [2]. Ursprünglich verfolgte dieses Gesetz gerade den gegenteiligen Zweck, nämlich die Abschaffung des Heilpraktikerberufs zur Zeit des Nationalsozialismus. Mit dem Verbot, Ausbildungsstätten für Heilpraktiker zu unterhalten, wollte man erreichen, dass keine neuen Heilpraktiker mehr ausgebildet werden konnten [3]; denn sowohl die heterogenen Strukturen des Heilpraktikerwesens als auch der liberale Grundsatz der Kurierfreiheit widersprachen den Prinzipien des autoritären Staats [4]. Nach Gründung der Bundesrepublik waren diese Regelungen allerdings nicht mehr verfassungskonform, denn sie standen im Widerspruch zum grundgesetzlich verankerten Prinzip der Freiheit der Berufswahl [5]. Gemäß $\S 1$ Abs. 1 HeilprG gilt in Deutschland seither ein generelles Berufsverbot mit Erlaubnisvorbehalt: Wer die Heilkunde ausüben will, ohne als Arzt bestallt zu sein, bedarf einer Erlaubnis. Diese darf dem Bewerber aber nicht versagt werden, wenn er alle persönlichen Voraussetzungen erfüllt, die in den zum HeilprG erlassenen Durchführungsverordnungen genannt sind [6]. Tabelle 1 zeigt diese in der Zusammenfassung. Somit enthält das HeilprG nunmehr sogar eine Anspruchsnorm auf Erlaubniserteilung zur Ausübung der heilberuflichen Tätigkeit bei Vorliegen der entsprechenden persönlichen Zulassungsvoraussetzungen [3]. Die Heilpraktikererlaubnis berechtigt ihren Inhaber grundsätzlich zur umfassenden Ausübung der Heilkunde [7] - mit einigen Ausnahmen, z.B. auf dem Gebiet der Zahnheilkunde oder der Geburtshilfe. (Anders als die einem Arzt mit Approbation erteilte Heilbefugnis ist die Heilpraktikererlaubnis teilbar. Derzeit kann eine beschränkte Erlaubnis für das Gebiet der Psychotherapie oder der Physiotherapie erlangt werden («kleiner Heilpraktiker») [7].) Zudem gibt es unter anderem Einschränkungen bezüglich verschreibungspflichtiger Arzneimittel oder bestimmter übertragbarer Krankheiten [4, 8].

Im Jahr 1960 waren in Deutschland gemäß Literaturangaben 2692 Heilpraktiker tätig, seither konnte jedoch ein starker Zuwachs beobachtet werden [9]. Die Anzahl der Heil- praktiker stieg von 13000 im Jahre 2000 auf 32000 im Jahre 2010. Im gleichen Zeitraum hat sich der Frauenanteil von $62 \%$ auf $72 \%$ erhöht [10]. Die Anzahl der selbstständigen Heilpraktiker wurde im Jahr 2007 auf ungefähr 8000-12 000 geschätzt, wovon ca. 5000 im Vollerwerb arbeiteten. Es ist davon auszugehen, dass zahlreiche zugelassene Heilpraktiker die Heilkunde nebenberuflich, z.B. in einer «Feierabend»Praxis ausüben [9]. Auch gibt es Hinweise darauf, dass Frauen beruflich relevantes Lernen in der Familienphase, z.B. zum Bestehen der Heilpraktikerprüfung, lediglich als «Hobby» bezeichnen [11]. Es wird angenommen, dass jedes Jahr etwa 15 Millionen Patientenkontakte in Heilpraktikerpraxen stattfinden. Jeder dritte Deutsche soll mindestens schon einmal einen Heilpraktiker aufgesucht haben [12].

Während die Kooperation zwischen Ärzten als Selbstverständlichkeit erachtet wird, gilt das Verhältnis zwischen Ärzten und Heilpraktikern als schwierig. Wünscht ein Patient die gemeinsame Behandlung durch einen Arzt und Heilpraktiker, ist dies nach den derzeitigen gesetzlichen Regelungen grundsätzlich nicht möglich. Die Berufsordnungen für Ärzte der jeweiligen Landesärztekammern enthalten Verbotsnormen, die dem $§ 29$ a Abs. 1 der Musterberufsordnung für Ärzte entsprechen. Diese untersagen Ärzten, zusammen mit Dritten, die weder Ärzte sind noch zu den berufsmäßig tätigen ärztlichen Mitarbeitern gehören, Patienten zu untersuchen oder zu behandeln. Zudem ist die Zusammenarbeit eines Heilpraktikers mit einem Arzt im Rahmen einer medizinischen Kooperation zwischen Ärzten und Angehörigen anderer Fachberufe im Gesundheitswesen gemäß $\S 23$ b Abs. 1 der Musterberufsordnung für Ärzte nicht vorgesehen und damit faktisch ausgeschlossen [3, 13].

Auch das Konkurrenzverhältnis der beiden Berufsgruppen zueinander kann eine effektive organisatorische oder fachliche Kooperation verhindern. Es gibt Hinweise darauf, dass komplementäre und alternative Heilverfahren, wie Akupunktur, Phytotherapie, Homöopathie oder Neuraltherapie, mittlerweile fester Bestandteil des ambulanten ärztlichen Angebots sind [14]. Durch Integration dieser Methoden in das Weiterbildungskonzept der Ärzte und die damit verbundene Möglichkeit, entsprechende Zusatzbezeichnungen zu erlangen (z.B. Akupunktur, Homöopathie oder Naturheilverfahren), wird darüber hinaus offensichtlich, dass Ärzte und Heilpraktiker im Marktsegment der komplementären und alternativen Heilverfahren zueinander im Wettbewerb stehen. Es ist davon auszugehen, dass berufspolitisch motivierte Forderun-
Tab. 1. Voraussetzungen für die Erteilung der Heilpraktikererlaubnis (eigene Darstellung nach [25])

\begin{tabular}{ll}
\hline Voraussetzungen & Nachweis wird erbracht durch \\
\hline Mindestalter 25 Jahre & Geburtsurkunde \\
Abgeschlossene Volksschulbildung & Abschlusszeugnis \\
Sittliche Zuverlässigkeit & polizeiliches Führungszeugnis \\
Gesundheitliche Eignung & ärtliches Attest \\
Keine Gefahr für die Volksgesundheit & Überprüfung durch das Gesundheitsamt \\
\hline
\end{tabular}


Tab. 2. Unterscheidungskriterien der Heilpraktikerschulen (eigene Darstellung nach [18])

\begin{tabular}{ll}
\hline Unterscheidungskriterium & Ausprägungen \\
\hline Ausbildungsmodelle & Präsenz- und Fernlehrgänge \\
& Vollzeit- und Teilzeitangebot \\
& reine Theorie- sowie kombinierte Theorie-Praxis-Ausbildungen \\
& Angebote für Heilpraktikeranwärter mit oder ohne medizinische Vorkenntnisse \\
& Angebote mit festem Klassengefüge bzw. mit flexiblem Einstieg in die Ausbildung \\
& (Rotationsprinzip) \\
\hline Ausbildungsinhalte & Konzentration auf die Vermittlung der - für die amtsärztliche Überprüfung relevanten - \\
& schulmedizinischen Inhalte \\
& zusätzliche Vermittlung von Kenntnissen zu komplementär- und alternativmedizinischen \\
& Heilverfahren, neben schulmedizinischen Inhalten \\
\hline Serviceleistungen & absolute Höhe und Fälligkeit der Kosten \\
& Teilnehmerzahl (maximale Kursgröße) \\
& Ermöglichung zur Teilnahme an einem Probeunterricht \\
\hline
\end{tabular}

gen seitens der Ärzteschaft, die Heilpraktikerschaft abzuschaffen, umso deutlicher werden, je erfolgreicher sich Heilpraktiker im Markt behaupten können [4]. So forderte beispielsweise der Deutsche Ärztetag den Gesetzgeber im Jahre 2008 auf, «das Heilpraktikerwesen endlich neu zu regeln! (...) Der Gesetzgeber muss sich endlich dazu bekennen, dass das Heilpraktikergesetz vom 17.02.1939 restlos veraltet ist und den Erfordernissen der medizinischen Versorgung der Bevölkerung in keiner Weise mehr entspricht» [15].

In der Europäischen Union bestehen (noch) große Unterschiede hinsichtlich der Zulassungsregeln für nichtärztliche Heiler. Eine Zulassung zum Heilpraktiker nach deutscher Rechtslage gibt es derzeit in keinem anderen Mitgliedsstaat. Deswegen fehlt auch die Grundlage der gegenseitigen Anerkennung. Will beispielsweise ein nach britischem Recht zugelassener Osteopath in Deutschland praktizieren, muss er ebenfalls die Heilpraktikererlaubnis erwerben und die Überprüfung durch den Amtsarzt bestehen [9]. Die Überprüfung der Heilpraktikeranwärter durch die Gesundheitsämter stellt jedoch keine allgemeine Fachprüfung dar, sondern dient lediglich der Gefahrenabwehr: Die Erlaubnis wird nicht erteilt, wenn sich aus einer Überprüfung der Kenntnisse und Fähigkeiten des Antragstellers durch das Gesundheitsamt ergibt, dass die Ausübung der Heilkunde durch den Betreffenden eine Gefahr für die Volksgesundheit bedeuten würde. Hintergrund der Bedenken gegenüber Heilpraktikern ist oftmals die Überlegung, dass die bloße Zulassung ohne angemessene Ausbildung nicht hinreichend für akzeptable Resultate bei der Versorgung von Patienten sein kann [16]. Kritisch hinterfragt wird dabei auch, dass bei der Überprüfung durch die Gesundheitsämter gerade diejenigen Methoden, die ein Heilpraktiker gemäß seinem Berufsverständnis bevorzugt anwenden wird, nämlich komplementäre und alternative Heilverfahren (Complementary and Alternative Medicine, CAM), nicht von Relevanz sind [6, 9]: «Heilpraktiker-Prüfungen decken ausschließlich schulmedizinisches Wissen ab, nicht das später gewählte Therapiegebiet» [17].
So ist es nicht verwunderlich, dass gerade die Ausbildung der Heilpraktiker im Zentrum der Kritik steht: Tatsächlich existieren keine Regelungen über Ausbildungsvoraussetzungen, Dauer oder Inhalt der Ausbildung [5]. Dem Heilpraktikeranwärter bleibt es damit freigestellt, ob er sich die Inhalte der amtsärztlichen Überprüfung im Selbststudium aneignet, Wochenendkurse absolviert oder sich zu einem mehrjährigen Besuch einer der zahlreichen Heilpraktikerschulen entschließt. Wie aus Tabelle 2 ersichtlich, ist deren Unterrichtsangebot hinsichtlich Ausbildungsmodellen und -inhalten sowie Ausbildungskosten und Serviceleistungen äußerst vielfältig [18]. Zunehmend lässt sich aber auch das Bestreben der Heilpraktikerverbände beobachten, eine fundierte Ausbildung des Nachwuchses durch verbandseigene Schulen sicherzustellen. Vorgesehen ist dabei beispielsweise ein mindestens 3-jähriger Lehrgang, der 3000 Unterrichtseinheiten zu jeweils 45 min umfasst. Zusätzlich ist eine praktische Ausbildung vorgesehen, die sich z.B. auf Blutdruckmessung, Palpation, Perkussion und Blutentnahmen erstreckt [6]. Anzumerken ist dabei, dass Heilpraktiker bzw. Heilpraktikeranwärter keinem Heilpraktikerverband beitreten müssen: Während die Ärzteschaft in Landesärztekammern als Körperschaften des öffentlichen Rechts mit Pflichtmitgliedschaften organisiert ist, werden die zahlreichen Heilpraktikerverbände als privatrechtliche Vereine mit freiwilliger Mitgliedschaft angesehen [3].

Kritisiert werden jedoch nicht nur die Zulassungsanforderungen für Heilpraktiker, sondern auch (vermeintliche) Ungleichbehandlungen bei der Berufsausübung. Während sich Ärzte in ihrer täglichen Arbeit stets wachsenden Anforderungen des Qualitätsmanagements ausgesetzt sehen, seien Qualitätsstandards für Heilpraktiker dagegen kaum existent [19]. Dabei darf jedoch nicht übersehen werden, dass zu den beruflichen Pflichten eines Heilpraktikers eine umfangreiche Rechtsprechung existiert. So sind an die Aufklärungspflicht eines Heilpraktikers grundsätzlich dieselben Maßstäbe anzulegen wie an die ärztliche Aufklärungspflicht. Darüber hinaus muss der Heilpraktiker mit seinen Patienten neben den medi- 
zinischen Fragen auch die finanzielle Tragweite der Behandlung erörtern. Ein Heilpraktiker, der invasive Methoden bei seinen Patienten anwendet, hat insoweit dieselben Sorgfaltspflichten zu erfüllen wie ein Arzt für Allgemeinmedizin - dies schließt auch die regelmäßige Fortbildung mit ein. Ebenso hat sich ein Heilpraktiker der Weiterbehandlung eines Patienten zu enthalten, wenn er bei der Behandlung an die Grenzen seiner therapeutischen Möglichkeiten stößt. In diesem Sinne hat der Heilpraktiker eine Zusammenarbeit mit Ärzten niemals zu unterlassen, sondern sogar jederzeit anzustreben [20].

Nach geltendem Recht wird er dabei allerdings auf Hindernisse stoßen, die in den Berufsordnungen der jeweiligen Landesärztekammern liegen. Im Gegensatz zu Ärzten ist Zahnärzten die Kooperation mit Heilpraktikern nicht untersagt. Dies wird damit begründet, dass der Heilpraktiker kraft des Gesetzes keine Zahnheilkunde ausüben darf und so eine genaue Abgrenzung der Behandlungsfelder von Heilpraktiker und Zahnarzt möglich sei. Vor dem Leitbild des mündigen Patienten kann daher nicht davon ausgegangen werden, dass es - anders als bei Ärzten - zu einer Verwirrung hinsichtlich beider Tätigkeitsbereiche kommt [3]. Offensichtlich geht die Rechtsprechung davon aus, dass die Öffentlichkeit nur unzureichend über Unterschiede in Ausbildung und Zulassung von Ärzten und Heilpraktikern informiert ist, sodass deren Berufs- und Verantwortungsgrenzen verschwimmen. Die Feststellung «in Germany, many patients can still not differentiate between physicians practising CAM and Heilpraktiker» [21] widerspricht der Vorstellung, dass Patienten eine rationale Entscheidung zwischen verschiedenen Anbietern von Gesundheitsdienstleistungen treffen können.

Unterschiedliche Ausbildungsstandards und Qualitätsanforderungen für Ärzte und Heilpraktiker werfen sowohl Fragen zur Patientensicherheit, aber auch bezüglich möglicher Wettbewerbsverzerrungen auf, denn Heilpraktikerbehandlungen stellen aus ökonomischer Sicht Konkurrenzangebote zur ärztlichen Versorgung dar: «For some patients, the Heilpraktiker already replaces the family doctor» [19]. Wenn dies auch nicht für das gesamte Behandlungsspektrum zutreffen mag, ist davon auszugehen, dass Ärzte und Heilpraktiker zumindest im Bereich komplementärer und alternativer Heilverfahren zueinander im Wettbewerb stehen [9]. So kann sich auch der Ratschlag eines Hausarztes an seine Kollegen erklären lassen, sich den positiven Einstellungen ihrer Patienten gegenüber der Naturheilkunde nicht zu verschließen, «sonst geht der Patient zum Heilpraktiker» [22]. Studien konnten zeigen, dass Patienten das größte Vertrauen in Heilpraktiker (61\%) als Informationslieferanten für alternative Heilverfahren setzen, während Ärzten und Apothekern (33\% und 29\%) diesbezüglich nur nachrangige Bedeutung beigemessen wird. Gleichzeitig sind $45 \%$ der Deutschen überzeugt, dass Ärzte die Möglichkeiten komplementärer und alternativer Heilverfahren zu wenig nutzen [23].

Die Zunahme der Heilpraktikerbehandlungen wird aber nicht nur mit einem Mangel an ärztlichen Angeboten komple- mentärer und alternativer Heilverfahren begründet: Patienten beklagen auch, dass Ärzte zu wenig Zeit für sie hätten, die Behandlung der Seele zu kurz komme und die schulmedizinische Betrachtung im Allgemeinen zu wenig «ganzheitlich» sei. Als Ursache dieser Defizite werden oftmals zeitliche - und damit letztlich wirtschaftliche - Zwänge genannt, denen Ärzte im System der gesetzlichen Krankenversicherung unterworfen seien [4, 17]. Dagegen besteht kein Leistungsanspruch gesetzlich Versicherter auf Behandlung durch einen Heilpraktiker. Diese stellt zunächst eine Selbstzahlerleistung dar. Jedoch können private Krankenversicherungen oder private Zusatzversicherungen der gesetzlichen Krankenkassen je nach vertraglicher Gestaltung die Kosten einer Behandlung durch den Heilpraktiker übernehmen [4]. Im Jahr 2009 gaben private Krankenversicherer 207,5 Millionen EUR für Heilpraktikerbehandlungen aus, dies entspricht einem Anteil von $2,2 \%$ der gesamten ambulanten Leistungsausgaben (zum Vergleich: ärztliche Behandlungen 55\%, Arzneien und Verbandmittel 25\%, Heilmittel 8,2\%, Hilfsmittel 7,9\%, Sonstiges $1,6 \%)$. Die Daten der privaten Krankenversicherung zeigen auch, dass mehr Frauen als Männer den Weg zum Heilpraktiker finden: Im Jahr 2009 waren rund 50,7\% der Behandelten weibliche Erwachsene, 33,7\% männliche Erwachsene und 15,6\% Kinder [24]. Aufgrund des wachsenden Wettbewerbs im Versicherungsmarkt ist damit zu rechnen, dass zukünftig immer mehr private Versicherer diese Option in ihr Angebot aufnehmen werden [25].

Studien konnten darüber hinaus zeigen, dass auch der Anteil der Bevölkerung, der komplementäre und alternative Heilverfahren in Anspruch nimmt, ansteigt [26]. Damit wächst auch der Anteil von Patienten, für die eine Heilpraktikerbehandlung attraktiv sein könnte. In Europa, Nordamerika und anderen industrialisierten Regionen haben bereits über 50\% der Bevölkerung CAM mindestens einmal in Anspruch genommen, in Entwicklungsländern ist von deutlich höheren Zahlen auszugehen. Während in Industrienationen CAM insbesondere von Wohlhabenden in Anspruch genommen wird, sind in ärmeren Regionen schulmedizinische Behandlungen für die Mehrheit der Bevölkerung oft schwer bezahl- oder erreichbar [27], jedoch sehen auch dort einige Autoren Defizite in der schulmedizinischen, westlich geprägten Arzt-Patienten-Beziehung als verantwortlich für die große Popularität der «traditional practitioners» [28].

Global lässt sich damit die Gemeinsamkeit feststellen, dass 2 (oder mehr) «Medizinsysteme» nebeneinander existieren. Diese Koexistenz wird dabei erst durch wahrgenommene Defizite des jeweils vorherrschenden Systems ermöglicht bzw. nötig. In Deutschland wird der Bedarf an medizinischer Grundversorgung zwar durch approbierte Ärzte abgedeckt, «dennoch scheint der Heilpraktiker insbesondere durch die Anwendung von Naturheilverfahren eine in der Bevölkerung empfundene Lücke gesundheitlicher Versorgung zu schließen» [9]. Aus Sicht der Gesundheitssystemforschung ist davon auszugehen, dass, selbst wenn Defizite auf der Mikro- 
Tab. 3. Beschreibung der Interviewpartner (eigene Darstellung)

\begin{tabular}{|c|c|}
\hline Kriterium & Ausprägung \\
\hline Fachgebiete & $\begin{array}{l}\text { Ärzte, } \mathrm{n}=15 \text {, davon auf folgenden Gebieten tätig: } \\
\text { Allgemeinmedizin, } \mathrm{n}=7 \\
\text { Haut- und Geschlechtskrankheiten, } \mathrm{n}=1 \\
\text { Frauenheilkunde und Geburtshilfe, } \mathrm{n}=3 \\
\text { Hals-Nasen-Ohrenheilkunde, } \mathrm{n}=1 \\
\text { Innere Medizin, } \mathrm{n}=2 \\
\text { Kinder- und Jugendmedizin, } \mathrm{n}=1 \\
\text { Zahnärzte, } \mathrm{n}=2\end{array}$ \\
\hline Alter & $\begin{array}{l}\text { Durchschnittsalter } 53 \text { Jahre } \\
\text { Altersspanne } 35-62 \text { Jahre }\end{array}$ \\
\hline Geschlecht & $\begin{array}{l}\text { männlich, } \mathrm{n}=13 \\
\text { weiblich, } \mathrm{n}=4\end{array}$ \\
\hline
\end{tabular}

ebene, also der Arzt-Patienten-Beziehung, wahrgenommen werden, die Ursachen auf der Systemebene lokalisiert sein können. Demnach werden insbesondere falsch gesetzte ökonomische Anreize für eine Vernachlässigung der «sprechenden Medizin» durch Schulmediziner verantwortlich gemacht. Zusätzlich kann jedoch die Frage gestellt werden, ob nicht gerade die medizinische Kooperation von Ärzten und Heilpraktikern ein Schritt hin zu mehr Patientenorientierung und -sicherheit in unserem Gesundheitssystem sein könnte [3]. Als ein mögliches Risiko der Heilpraktikerbehandlung gilt, dass infolge ungeeigneter Diagnoseverfahren Erkrankungen nicht rechtzeitig erkannt bzw. unangemessene Therapieverfahren zu lange vergeblich angewendet werden [29]. Diese Problematik könnte eventuell durch Zulassung und Förderung von Kooperationsmöglichkeiten zwischen Ärzten und Heilpraktikern entschärft werden. Zudem ist es denkbar, dass aus Patientensicht ein Bedarf an entsprechenden Kooperationsangeboten besteht. Falls die Synergieeffekte von Arzt und Heilpraktiker für Patienten tatsächlich von hohem therapeutischen Nutzen sind [4], sollten die Potenziale der unterschiedlichen Berufsgruppen für eine effektive und effiziente Gesundheitsversorgung genutzt werden. Dies setzt jedoch bei allen Gesundheitsberufen zuallererst die Bereitschaft voraus, zu einer Aufteilung der Tätigkeitsfelder und zu neuen, teamorientierten Arbeitsformen zu kommen [5].

\section{Methodik}

Basierend auf der Literaturrecherche wurde ein Gesprächsleitfaden entwickelt, der bei 15 niedergelassenen Ärzten und 2 Zahnärzten zur Anwendung kam. Es handelte sich um eine teilstrukturierte Interviewsituation, wobei ausschließlich offene Fragen verwendet wurden. Der Gesprächsleitfaden wurde während der Erhebungsphase responsiv weiterentwickelt: Während der Interviews gewonnene Erfahrungen ließen sich sukzessive in den folgenden Gesprächen nutzen, um die Leitfragen zu spezifizieren. Wenn Befragte ein Thema ansprachen, das im Leitfaden erst später vorgesehen war, wurde es vorgezogen. Als Auswahlstrategie für die Interviewpartner wurde das theoretische Sampling gewählt [30].
Das Erreichen von Repräsentativität war gemäß den Kriterien der qualitativen Forschung kein Ziel bei der Auswahl der Interviewpartner, sondern deren erwarteter Beitrag zur Theoriegenerierung. Dazu sollten nicht nur ähnliche, sondern auch widersprüchliche Daten (kontrastierende Beispiele) herangezogen werden [31]. Tabelle 3 beschreibt die Interviewpartner nach Fachgebiet, Alter und Geschlecht. Die Rekrutierung wurde fortgesetzt, bis genügend Teilnehmer akquiriert worden waren.

Nach Zustimmung der Interviewpartner wurden die Gespräche auf Tonband aufgezeichnet. Zusätzlich wurden unmittelbar nach dem Gespräch Postskripte erstellt. Sie enthielten demographische Angaben des Interviewpartners, Anmerkungen zu situativen und nonverbalen Aspekten sowie zu Schwerpunktsetzungen des Forschungsgesprächs. Außerdem wurden spontane thematische Auffälligkeiten und Interpretationsideen notiert, die Anregungen für die Auswertung geben konnten. Folgende Fragestellungen flossen in die Gespräche ein:

- Besteht bei den befragten Ärzten grundsätzlich die Bereitschaft zur Zusammenarbeit mit Heilpraktikern?

- Welche Bedingungen werden an die Zusammenarbeit gestellt?

- Welche Chancen könnten sich aus einer Kooperation ergeben?

- Welche Hindernisse gibt es, die einer Zusammenarbeit entgegenstehen?

Im Anschluss wurden die Interviews wörtlich transkribiert und mithilfe der thematischen Inhaltsanalyse ausgewertet [32]. Dabei wurde der Text zunächst mit Stichworten aus dem Leitfaden (deduktiv) und mit Begrifflichkeiten, die neue thematische Aspekte aus den Darstellungen der Interviewpartner kennzeichneten (induktiv), markiert. Diese Markierungen bildeten die Basis für die Entwicklung eines Kodierrasters, mithilfe dessen die einzelnen Aussagen der Transkripte bestimmten Kategorien zugeordnet wurden. Die Kategorien wurden primär anhand der Themenbereiche entwickelt, die in den Interviews systematisch abgefragt wurden, um dann im Laufe der Analyse durch Aspekte, die sich in den Interviews neu ergeben hatten, erweitert zu werden [33]. Die Transkripte wurden vor und nach dem Kodieren von 2 der Autoren wiederholt gelesen, um die Kategorisierung der Daten zu überprüfen. Nach Erstellung einer Textdatenbank konnte diese als elektronisches Fundstellenregister genutzt werden

\section{Ergebnisse}

\section{Grundsätzliche Bereitschaft zur Zusammenarbeit}

Die Befragung zeigte eine grundsätzliche Aufgeschlossenheit gegenüber einer Kooperation mit Heilpraktikern: Wenn es Behandlungserfolg verspräche, insbesondere wenn sich durch schulmedizinische Therapie allein nur unbefriedigende Ergebnisse erzielen lassen, sei Konkurrenzdenken fehl am Platz. Heilpraktiker sollten dann ergänzende Therapeuten als Teil eines Therapienetzwerkes sein. Dadurch lasse sich z.B. das Risiko vermindern, dass schwere Krankheiten übersehen werden.

- «(Ob ich mir eine Zusammenarbeit mit Heilpraktikern vorstellen kann?) Also, ich bin da absolut frei von Berührungsängsten».

- «Sofern sich (meine und die Behandlung des Heilpraktikers) ergänzen würden, dann hätte ich keine Bedenken».

- «(Eine Zusammenarbeit mit Heilpraktikern) ist zumindest schwierig. Es ist nicht undenkbar».

- «(Einer Zusammenarbeit mit Heilpraktikern stehe ich aufgeschlossen gegenüber,) weil ich denke: Wer heilt, hat Recht, 
vollkommen egal, wie er heilt. Es gibt ja auch viele Sachen, die sehr gut sind. Und das ist eine gute Kombination».

- «Das ist für mich eben bezeichnend, dass ich durchaus die Heilpraktiker nicht als Konkurrenzunternehmen sehe, sondern eben auch einfach als ein Rädchen im ganzen Räderwerk des Diagnostik- und Therapieerfolgs».

- «Ich könnte mir das durchaus vorstellen. Ich bin sehr offen. Ich bin kein engstirniger und scheuklappenmäßiger Schulmediziner - auch nicht unbedingt nach dem Motto, gut ist, was hilft - aber es ist schon etwas daran an diesem Sprichwort. Und ich könnte mir eine Zusammenarbeit durchaus vorstellen».

- «Das Ideale wäre, der Heilpraktiker soll der ergänzende Therapeut sein, wenn die Patientenstruktur hierfür geeignet ist und wenn die schulmedizinischen Behandlungsmethoden zwar nicht abgebrochen werden, aber für sich alleine keinen ausreichend befriedigenden Erfolg bei diesem Patienten gezeigt haben».

- «Und wenn einer einen Wunsch hat, zu einem Heilpraktiker zu gehen, und mich deswegen fragt, dann sag ich ihm immer, dass ich nichts dagegen habe. Ich würde aber gerne wissen, was er tut, weil ich nur den Wunsch habe, dass ihm nicht geschadet wird oder dass nichts übersehen wird. Aber ansonsten kann man das sehr offen diskutieren».

Allerdings konnte auch ein kontrastierendes Beispiel gefunden werden, denn ein Interviewpartner schloss für sich die Zusammenarbeit mit Heilpraktikern gänzlich aus. Diese arbeiteten auf einem völlig anderen Gebiet als Ärzte und könnten im Rahmen ihrer Möglichkeiten, die auch spiritueller Art seien, schwere Erkrankungen nicht heilen.

- «Ich würde aber nie zu einem Heilpraktiker überweisen. (... Eine Zusammenarbeit wäre für mich undenkbar,) weil der Heilpraktiker auf einem komplett anderen Gebiet arbeitet als der Arzt. Der Heilpraktiker selbst kann eine schwere Erkrankung weder behandeln noch heilen, weil er es gar nicht darf und weil er es auch nicht kann mit den Mitteln, die ihm zur Verfügung stehen. Da geht es dann in das Spirituelle herein».

\section{Bedingungen für eine Zusammenarbeit}

Die Interviews machten deutlich, dass eine Kooperation an bestimmte Voraussetzungen geknüpft wird: das Erkennen der Grenzen alternativer Behandlungen durch den Heilpraktiker, die ärztliche Überzeugung von der Wirksamkeit des angebotenen Verfahrens und ein Mangel an ärztlichen Angeboten in diesem Bereich.

- «Es gibt da gar kein Problem, wenn jeder seine Grenzen kennt und weiß, wo er gut ist und wo er schlecht ist - gar kein Problem. Der Heilpraktiker muss genau wissen: Aha, da muss ich jetzt aufpassen».

- «(Eine Zusammenarbeit käme nur unter folgenden Bedingungen infrage:) Der Betreffende, der hier dann als Heil- praktiker tätig ist, der müsste tatsächlich auf dem Teppich bleiben und müsste sagen, er macht also z.B. 3 oder 4 Behandlungen und dann versucht er das oder jenes - und wenn er dann nicht zurechtkommt, müsste zumindest eine schulärztliche oder fachärztliche Behandlung folgen».

- «Wenn ich davon überzeugt bin, dass jemand bestimmte Dinge macht als Heilpraktiker, Dinge, die die meisten Mediziner nicht machen, und ich weiß, dass diese Methode, die er da macht, durchaus erfolgreich sein kann, dann hätte ich überhaupt nichts dagegen, dass ich ihn dorthin schicke».

Betont wurde auch die Notwendigkeit von Transparenz bezüglich der Vergütung und Kostenerstattung von jeweils erbrachten Leistungen. Obwohl die Heilpraktikerbehandlung keine Leistung der gesetzlichen Krankenversicherung darstellt, scheint hinsichtlich der Kostenerstattung alternativer Behandlungsverfahren dennoch eine ärztliche Benachteiligung wahrgenommen zu werden: Die einseitige Abdeckung von Heilpraktikerbehandlungen durch private Kranken- oder Zusatzversicherungen führe $\mathrm{zu}$ einer Diskriminierung des ärztlichen CAM-Angebots, z.B. der homöopathischen Behandlung durch Ärzte.

- «Heilpraktiker sind erst mal die erste Anlaufstelle für viele, weil es krankenversicherungstechnisch häufig bezahlt wird. Heilpraktikerbehandlung wird häufiger bezahlt als die homöopathische Behandlung bei Ärzten... Ich habe eine ganze Menge Patienten, die mich bitten, nach der Heilpraktiker-Gebührenordnung abzurechnen, weil sie es dann erstattet kriegen. Und das kann ich nicht, weil auch durch meinen Doktortitel auffällt, dass ich Arzt bin».

\section{Chancen einer Zusammenarbeit}

Einer Zusammenarbeit gegenüber aufgeschlossene Ärzte sehen Heilpraktiker als ergänzende Therapeuten bei leichteren oder psychisch beeinflussten Erkrankungen an.

- «Beim Heilpraktiker habe ich nur dann ein Problem, wenn der Sachen behandelt, die mit diesen Methoden sicher nicht behandelbar sind, oder wenn er sich in Dinge einmischt, wo er einfach nichts zu suchen hat. Wenn es also wirklich um sehr schwere Krankheiten geht, da hat der Heilpraktiker mit seinen Methoden nichts zu suchen».

- «Im Grunde genommen ist das ja eine Psychotherapie, wenn ein Heilpraktiker durch Vertrauen den Patienten zum Erfolg verhilft. Eine ernste Krankheit kann er nicht heilen... Und deswegen hat er auch eine Berechtigung, weil in der Schulmedizin leider die Psychotherapie zu wenig Wirkung zeigen kann, weil es zu selten angewendet wird und weil es auch schlecht vergütet wird».

- «Ich finde es schon erstaunlich, dass viele Menschen zum Heilpraktiker gehen und sich hinterher bei ihm besser betreut fühlen als beim Arzt. Das gibt es ja. Es gibt immer wieder Patienten, die herkommen und sagen: Ja, und dann 
bin ich zum Heilpraktiker gegangen, und es war weg. Das sind aber auch viele Beschwerden, muss man sagen, die nicht somatischer Natur sind. Und da hat der Heilpraktiker vielleicht sein Recht».

- «(Eine Zusammenarbeit kann ich mir schon vorstellen,) z.B. dass die Psyche eher von den Alternativmedizinern oder von den Heilpraktikern behandelt wird. Und das andere, schwerwiegende Geschichten - auch operative - von den Schulmedizinern».

\section{Hindernisse einer Zusammenarbeit}

Aus den Interviews ergaben sich konkrete Hinweise auf bestehende Barrieren für eine Zusammenarbeit von Ärzten und Heilpraktikern, z.B. das mangelnde Vertrauen in die Ausbildung der Heilpraktiker. Zudem wurde deutlich, dass offensichtlich auch Patienten diese Hindernisse wahrnehmen. Darauf weist beispielsweise die Formulierung hin, Patienten müssten «zugeben» oder «sich dafür entschuldigen», beim Heilpraktiker gewesen zu sein. Bereits diese Wortwahl lässt auf eine Voreingenommenheit schließen, die eine offene Arzt-Patienten-Kommunikation über Heilpraktikerbesuche eher behindern dürfte.

- «Also ich habe sehr viele Patientinnen, die mir gegenüber dann zugeben, und die sagen das auch freiwillig: Herr Doktor, ich war jetzt beim Heilpraktiker».

- «Es ist oft so im Nebensatz, so entschuldigend: Und ich gehe übrigens auch noch zum Heilpraktiker».

- «Viele denken ja immer, ich bin böse, wenn man es mir sagt (dass man als Patient beim Heilpraktiker war). Ich bin nicht böse, nein».

\section{Mangelndes Vertrauen der Ärzte in die Heilpraktiker- ausbildung}

Aus den Interviews ging hervor, dass sich das fehlende Vertrauen der befragten Ärzte in die Heilpraktikerausbildung hemmend auf eine Zusammenarbeit auswirken kann. Eine fehlende Qualifikation wird auch in engem Zusammenhang dazu gesehen, dass Heilpraktiker gegebenenfalls die Grenzen ihrer Behandlungsfähigkeit nicht erkennen können. Einschränkend wurde allerdings festgestellt, dass auch eine fundierte Ausbildung keine Garantie dafür sei, denn Überschätzung der eigenen Kenntnisse und Fähigkeiten könne auch bei Ärzten vorkommen - trotz des Medizinstudiums.

- «(Eine Zusammenarbeit mit Heilpraktikern sehe ich schon problematisch,) weil die Ausbildung des Heilpraktikers doch anders ist als die Ausbildung des Mediziners und ich deswegen ein schlechtes Gefühl habe, weil ich denke, dass der im Endeffekt ein Laie bleibt, der Heilpraktiker».

- «Da der Heilpraktiker doch im Endeffekt ein medizinisch gebildeter Laie ist, sieht der oft seine Grenzen nicht. Das gibt es natürlich in der Schulmedizin genauso, dass einer alles kann und dann doch nicht alles kann. Aber ein Heil- praktiker wird wahrscheinlich manchmal seine Grenzen überschreiten, und dann kann er natürlich bei einer ernsten Krankheit schon Schaden anrichten - erheblichen Schaden anrichten».

Das mangelnde Systemvertrauen in die Ausbildung des Heilpraktikers kann dazu führen, dass eine Zusammenarbeit eher mit naturheilkundlich spezialisierten Ärzten gesucht wird oder sich die Bereitschaft zur Zusammenarbeit nicht auf den gesamten Berufsstand der Heilpraktiker bezieht, sondern nur auf einzelne Heilpraktiker, deren individuelle Vorgehensweisen und Kompetenzen man selbst einschätzen kann.

- «Aber das sind dann eher Kollegen, die sich naturheilkundlich spezialisiert haben, als dass ich z. B. einen Heilpraktiker empfehlen würde. Die kennt man dann nämlich doch nicht so sehr. Also ich zumindest nicht, und auch vom Hörensagen glaube ich, dass das noch einmal einen Schritt weiter weg ist».

- «Geh mal zum Heilpraktiker und lass das auspendeln! Ohne zu wissen, was der eigentlich tut - das würde ich nicht tun. Sondern ich sollte ein bisschen eine Ahnung haben, individuell, was treibt der, kann der dem Patienten, den ich jetzt da habe, weiterhelfen. Wenn das so ist, dann selbstverständlich».

\section{Sorge vor Beeinträchtigung der schulmedizinischen Therapie} Als weiteres Hindernis für eine Zusammenarbeit nannten die befragten Ärzte ihre Sorge darüber, dass Heilpraktiker schulmedizinische Diagnosen und Therapien gänzlich infrage stellen. Dies könne beispielsweise dazu führen, dass Patienten ihre gewohnten Medikamente absetzen oder das Vertrauen in die ärztliche Therapie verlieren. Beides wirke sich auch negativ auf die Arzt-Patienten-Beziehung aus.

- «Ich denke, der Heilpraktiker muss einfach wissen, wann schulmedizinisch etwas notwendig ist. Er darf einfach dieses ausschließliche Denken nicht haben. Ich meine sonst, der Patient wird nicht mehr losgelassen».

- «Aber wenn die (Heilpraktiker) jetzt Sachen absetzen, die lebensnotwendig sind, dann hätte ich schon Bedenken».

- «Und letztendlich versucht (der Heilpraktiker) die Patienten auch so ein bisschen abzuhalten von dem, was es bei uns gibt: Sie dürfen kein Kortison nehmen und so weiter und so fort. Das ist ja immer ganz einfach. Sie verteufeln sozusagen immer uns Schulmediziner, die ja nur die harten Sachen machen».

- «Die (Heilpraktiker) können unter Umständen ganz schöne Störfälle darstellen. Weil die (Patienten) kommen dann mit irgendeiner Auffassung und sagen: Ja, aber mein Heilpraktiker oder der und der hat gesagt, ich darf jetzt das und das überhaupt nicht mehr machen. Und dann kommt eben irgendwas dabei heraus, was den Behandlungsfluss hemmt. Und auch wenn man dann mit eindeutigen Diagnosen kommt, sei es röntgenologisch oder labormäßig, dann sträuben sie sich, diese Diagnosen eben auch $\mathrm{zu}$ akzeptieren». 
Tab. 4. Bedingungen, Chancen und Hindernisse einer Zusammenarbeit zwischen Ärzten und Heilpraktikern aus ärztlicher Sicht (eigene Darstellung)

\begin{tabular}{ll}
\hline Bedingungen für eine Zusammenarbeit & $\begin{array}{l}\text { Heilpraktiker erkennt die Grenzen seiner Behandlungsfähigkeit } \\
\text { ärztliche Überzeugung von der Wirksamkeit des angebotenen Verfahrens } \\
\text { mangelndes ärztliches Angebot in diesem Bereich } \\
\text { transparente Regelungen für Vergütung und Kostenerstattung ohne } \\
\text { Diskriminierung ärztlicher Leistungen }\end{array}$ \\
\hline Chancen einer Zusammenarbeit & $\begin{array}{l}\text { Heilpraktiker als ergänzender Therapeut bei } \\
\text { leichteren Erkrankungen } \\
\text { psychisch beeinflussten Erkrankungen } \\
\text { Erkrankungen, die sich schulmedizinisch nicht ausreichend } \\
\text { behandelbar zeigen }\end{array}$ \\
\hline Hindernisse einer Zusammenarbeit & $\begin{array}{l}\text { Kommunikationsdefizite zwischen Ärzten und Patienten hinsichtlich } \\
\text { einer gleichzeitigen Heilpraktikerbehandlung } \\
\text { mangelndes Vertrauen der Ärzte in die Heilpraktikerausbildung } \\
\text { Sorge vor Beeinträchtigung der schulmedizinischen Therapie } \\
\text { Erhöhung der Komplexität des Versorgungsalltags }\end{array}$ \\
\hline
\end{tabular}

\section{Erhöhung der Komplexität des Versorgungsalltags}

Auch wegen der bereits bestehenden Komplexität des Versorgungsablaufs wird eine Zusammenarbeit mit Heilpraktikern als schwierig erachtet. Schon jetzt sei es fast unmöglich, alle ärztlichen Leitlinien und Anforderungen an das Qualitätsmanagement zu berücksichtigen. Zusätzlich Heilpraktiker in den Versorgungsablauf zu integrieren, würde darüber hinaus einen erheblichen Mehraufwand für die Koordination der Teilprozesse bedeuten.

- «(Eine Zusammenarbeit mit Heilpraktikern?) Also ich muss ganz ehrlich sagen, das halte ich für etwas schwierig. Denn hier bei mir sind so viele Fakten und Laborwerte und Hintergründe mit Therapieschemata und mit Qualitätsmanagement und Leitlinien bzw. Richtlinien zu beachten, und wenn da zu diesem ganzen Zeug noch jemand hinzukommt ...»

\section{Diskussion und Schlussfolgerungen}

Die befragten Ärzte standen einer Zusammenarbeit mit Heilpraktikern grundsätzlich offen gegenüber. Es wurde jedoch deutlich, dass eine Kooperation an klare Bedingungen geknüpft ist und derzeit noch zahlreiche Hindernisse dafür existieren:

Ärzte, die einer Zusammenarbeit aufgeschlossen gegenüberstehen, sehen Heilpraktiker als ergänzende Therapeuten bei leichteren oder psychisch beeinflussten Erkrankungen. Heilpraktiker müssten allerdings klar die Grenzen ihrer Behandlungsfähigkeit erkennen. Voraussetzungen seien auch transparente Regelungen für Vergütung und Kostenerstattung durch Krankenkassen; insbesondere dürften ärztliche Leistungen im Bereich der Komplementär- und Alternativmedizin nicht diskriminiert werden. Es zeigte sich, dass die derzeitigen Ausbildungsstrukturen für Heilpraktiker es anscheinend nicht ermöglichen, bei Ärzten ein dem Medizinstu- dium vergleichbares Systemvertrauen zu erzeugen. Analog rät beispielweise auch die Stiftung Warentest, Patienten sollten bei der Auswahl des Heilpraktikers Vorsicht walten lassen: «Vertrauen Sie nicht jedem, der seine Heilkunst anbietet» [17]. Daneben erweist es sich auch als hinderlich, wenn Heilpraktiker störend auf die bestehende Arzt-PatientenBeziehung einwirken bzw. den Abbruch einer schulmedizinischen Therapie veranlassen. Gleichzeitig wird die Patientenversorgung auch ohne Integration von Heilpraktikern bereits als sehr komplex eingeschätzt, sodass eine Zusammenarbeit als schwierig erachtet wird. Tabelle 4 zeigt die Ergebnisse in der Zusammenfassung.

Ein Befragter hatte die Zusammenarbeit mit Heilpraktikern für sich gänzlich ausgeschlossen (kontrastierendes Beispiel), da deren Art zu heilen spirituelle Ansätze zeige. Auch in der Literatur liest man zu den Berufsinhalten des Heilpraktikers: «Seine Tätigkeit basiert auf Kenntnissen und Erfahrungen aus der Naturheilkunde (...) sowie aus überlieferten und bewährten Methoden der Volksheilkunde. Hinzu kommen neuere Diagnose- und Therapieverfahren, sowie auch einige esoterische Verfahren» [9]. Allerdings muss eine generelle Gleichsetzung von Heilpraktikern und Geistheilern wohl verneint werden: Gemäß Bundesverwaltungsgericht erweckt ein Heiler, der spirituell wirkt und den religiösen Riten näher steht als der Medizin, die Erwartung auf heilkundlichen Beistand erst gar nicht. Die Gefahr, notwendige ärztliche Hilfe zu versäumen, werde daher eher vergrößert, wenn geistiges Heilen als Teil der Berufsausübung von Heilpraktikern verstanden wird [20]. Trotzdem scheint es aber innerhalb der Ärzteschaft bezüglich der Fragestellung, inwieweit die Heilmethoden eines Heilpraktikers «spiritueller» oder «esoterischer» Art seien, Wahrnehmungsunterschiede zu geben, vergleichbar mit der Diskussion um die Akzeptanz einzelner Verfahren innerhalb des komplementären und alternativen Spektrums: «Die Bandbreite reicht von der Bachblüten-Therapie über Homöopathika bis hin zu Phytopharmaka. Für die einen, 
darunter auch die Arzneimittelkommission der deutschen Ärzteschaft (AkdÄ), fängt die Esoterik bereits bei Phytopharmaka an» [34]. Gegebenenfalls könnten hier Forschungskooperationen mit Vertretern der Religionswissenschaften zu Begriffsklärungen beitragen.

Um zu reproduzierbaren Ergebnissen bezüglich der ärztlichen Bereitschaft zur Zusammenarbeit mit Heilpraktikern zu gelangen, sind Untersuchungen mit quantitativem Forschungsdesign nötig. Auch sollte geklärt werden, inwieweit aus Sicht der Heilpraktiker eine Zusammenarbeit mit Ärzten wünschenswert ist. Danach kann das Themenfeld einer vertieften qualitativen Analyse unterzogen werden, um eine Konkretisierung des Begriffs «Zusammenarbeit» zu erreichen. Denkbar wäre etwa, anhand definierter Krankheitsbilder die Möglichkeiten einer Kooperation exemplarisch auszuloten. Um zu verlässlichen Aussagen zur Effektivität oder Effizienz einer Zusammenarbeit von Ärzten und Heilpraktikern zu gelangen, müsste beteiligten Ärzten von den Landesärztekammern zumindest für diesbezügliche Modellprojekte Raum gegeben werden. In jedem Fall erscheinen interdisziplinäre Forschungsansätze unter Einbezug der Rechtswissenschaften ratsam.

Für die Praxis kann derzeit die Schlussfolgerung gezogen werden, dass Kommunikationsbarrieren zwischen Ärzten und Patienten abgebaut werden sollten, damit Patienten offen mit ihrem Arzt über Heilpraktikerbesuche sprechen können. Die
Befunde unterstreichen die an Ärzte herangetragene Minimalforderung, Patienten explizit nach einem Heilpraktikerbesuch bzw. der Inanspruchnahme von komplementären und alternativen Heilverfahren zu fragen - auch im Falle der eigenen generellen Ablehnung dieser Methoden bzw. deren Ausübung durch nichtärztliche Heiler: «Ideally, GPs should routinely ask patients about CAM use in order to reduce the incidence of 〈complementogenic〉 disease» [35]. Insofern können auch Ärzte einen Beitrag dazu leisten, dass Patienten rechtzeitig eine gegebenenfalls notwendige schulmedizinische Therapie erhalten und mögliche Wechselwirkungen mit Therapien, die der Heilpraktiker empfiehlt, beachtet werden.

\section{Dank}

Die vorgestellte Befragung ist Teil des von der Deutschen Forschungsgemeinschaft (DFG) finanzierten Projekts «Das Phänomen der ärztlichen Hinwendung zu komplementären und alternativen Heilverfahren - Motive und Perspektiven im Spannungsfeld von Ökonomie und ärztlichem Selbstverständnis: Eine gesundheitswissenschaftliche Betrachtung» (NA 184/8-1).

\section{Disclosure Statement}

Die Autoren geben an, dass keine Interessenkonflikte bestehen.

\section{Literatur}

1 Dannecker G: Alles was Recht ist. Alternative Heilverfahren in rechtlicher Sicht; in Ritter WH, Wolf B (eds): Heilung - Energie - Geist. Heilung zwischen Wissenschaft, Religion und Geschäft. Göttingen, Vandenhoeck \& Ruprecht, 2005, pp 152-186.

2 Gesetz über die berufsmäßige Ausübung der Heilkunde ohne Bestallung (Heilpraktikergesetz), ausgefertigt am 17.02.1939, zuletzt geändert durch Artikel 15 des Gesetzes vom 23. Oktober 2001 (BGBl. I S. 2702).

3 Walburg I: Die Zusammenarbeit von Ärzten und Heilpraktikern nach den ärztlichen Berufsordnungen im Lichte der Berufsfreiheit; in Becker R, Sertel S, Stassen-Rapp I, Walburg I (eds): «Neue» Wege in der Medizin. Alternativmedizin - Fluch oder Segen? Heidelberg, Winter, 2010, pp 297-319.

4 Sasse R: Der Heilpraktiker. Ein Gesundheitsberuf ohne Berufsausübungsrecht? Dortmund, Nomos, 2011, pp 20, 21, 34, 35, 111, 108, 109, 213

5 Sachverständigenrat zur Begutachtung der Entwicklung im Gesundheitswesen: Kooperation und Verantwortung - Voraussetzungen einer zielorientierten Gesundheitsversorgung (Gutachten). Köln, Bundesanzeiger Verlagsgesellschaft, 2007, pp 26, 47, 57.

6 Tamm B: Die Zulässigkeit von Außenseitermethoden und die dabei zu beachtenden Sorgfaltspflichten. Berlin, Duncker \& Humblot, 2007, pp 63, 92, 93.

7 Berner B: Beschränkte Heilpraktikererlaubnis ist zulässig. Dtsch Arztebl 2009;106:A2527.

8 Robert Koch-Institut: Gesundheitsberichterstattung des Bundes, Heft 9. Inanspruchnahme alternativer Methoden in der Medizin. Berlin, 2002.
9 Engler B, Donhauser H: Wege zum Heilpraktiker. Stuttgart, Aktion Bildungsinformation, 2007, pp $25,28,32$.

10 Statistisches Bundesamt: Gesundheitspersonal. www.destatis.de/DE/ZahlenFakten/GesellschaftStaat/Gesundheit/Gesundheitspersonal/Tabellen/ Berufe.html (Zugriff am 18.07.2012).

11 Schaffert S: Beruflich relevantes Lernen von Frauen in der Familienphase. Empirische Analysen zu den Lernaktivitäten von Müttern während der familienbedingten Berufsunterbrechung. Dissertation LMU München, 2007, p 206.

12 Ernst E: Heilpraktiker in Österreich? Wien Med Wochenschr 1997;147:423-425.

13 (Muster-)Berufsordnung für die in Deutschland tätigen Ärztinnen und Ärzte, MBO-Ä 1997, in der Fassung der Beschlüsse des 114. Deutschen Ärztetages 2011 in Kiel.

14 Thanner M, Loss J, Nagel E: Komplementäre und alternative Heilverfahren im niedergelassenen Bereich: Ausmaß und Spektrum des ärztlichen Angebots. Gesundheitswesen 2011;73:621-622.

15 Bundesärztekammer: Beschlussprotokoll des 111. Deutschen Ärztetages vom 20. bis 23. Mai 2008 in Ulm. www.bundesaerztekammer.de/page.asp?his = 0.2.20.5711.6205 (Zugriff am 18.07.2012).

16 Ernst E: Complementary Medicine. Arch Intern Med 1995:155:2405-2408.

17 Stiftung Warentest: Mit Nadel und Globuli. Heilpraktiker, 2008. www.test.de/Heilpraktiker-Gesamteindruck-positiv-1652630-2652630/ (Zugriff am 18.07.2012).
18 Plöthner M, Thanner M, Nagel E: Heilpraktikerschulen im Vergleich: Ergebnisse einer explorativen Analyse. Gesundheitswesen 2011;73:598.

19 Joos S, Musselmann B, Szecsenyi J: Integration of complementary and alternative medicine into family practices in Germany: results of a national survey. Evid Based Complement Alternat Med 2011;2011:495813.

20 Stahl-Kadlec C, Donhauser H: Rechts- und Berufskunde für Heilpraktiker und Heilpraktikeranwärter. Berlin, Lehmanns Media, 2008, pp 22, 24-28, 113.

-21 Joos S, Musselmann B, Miksch A, Rosemann T, Szecsenyi J: The role of complementary and alternative medicine (CAM) in Germany - a focus group study of GPs. BMC Health Serv Res 2008;8:127.

22 Einecke U: Überlassen Sie das Feld nicht dem Heilpraktiker. MMW Fortschr Med 2008;150:1416.

23 Bühring P: Ganzheitliche Therapie gewünscht Dtsch Arztebl 2001;98:A1307.

24 Verband der privaten Krankenversicherung: Zahlenbericht der privaten Krankenversicherung 2009/2010. Korrigierte Version (Stand Januar 2011), pp 53, 56

25 Richter I: Lehrbuch für Heilpraktiker. Medizinische und juristische Fakten. München, Urban \& Fischer, 2004, pp 5, 47.

26 Eisenberg DM, Davis RB, Ettner SL, Appel S, Wilkey S, Van Rompay M, Kessler RC: Trends in alternative medicine use in the United States, 1990-1997: result of a follow-up national survey. JAMA 1998;280:1569-1575. 
27 World Health Organization: WHO Traditional Medicine Strategy 2002-2005. Geneva, WHO, 2002.

28 Lebhardt ND, Aboa SM, Manga E, Bensing JM, Langewitz W: Bridging the gap: how traditional healers interact with their patients. A comparative study in Cameroon. Trop Med Int Health 2010; 15:1099-1108.

29 Heudorf U, Carstens A, Exner M: Heilpraktiker und öffentliches Gesundheitswesen. Bundesgesundheitsblatt 2010;53:245-257.
30 Glaser BG, Strauss AL: Grounded Theory. Strategien qualitativer Forschung. Bern, Hans Huber, 2005, pp 53.

31 Mays N, Pope C: Qualitative research in health care. Assessing quality in qualitative research. BMJ 2000;320:50-52.

32 Froschauer U, Lueger M: Das qualitative Interview. Zur Praxis interpretativer Analyse sozialer Systeme. Wien, WUV, 2003.
33 Bortz J, Döring N: Forschungsmethoden und Evaluation für Human- und Sozialwissenschaftler. Heidelberg, Springer, 2006, pp 330.

34 Korzilius H: «Alternative Heilmethoden». Eine Art Glaubenskrieg. Dtsch Arztebl 1998;95:A2075A2079.

35 Schmidt K, Jacobs PA, Barton A: Cross-cultural differences in GPs' attitudes towards complementary and alternative medicine: a survey comparing regions of the UK and Germany. Complement Ther Med 2002;10:141-147. 